\title{
BMJ Open Status migrainosus inpatient treatment with eptinezumab (SMITE): study protocol for a randomised controlled trial
}

\author{
Jason Charles Ray (10 , ${ }^{1,2,3}$ Zhibin Chen, ${ }^{3}$ Georgia Ramsay, ${ }^{1}$ Jack Germaine, ${ }^{1}$ \\ Elspeth J Hutton ${ }^{1,3}$
}

To cite: Ray JC, Chen Z,

Ramsay G, et al. Status migrainosus inpatient treatment with eptinezumab (SMITE): study protocol for a randomised controlled trial. BMJ Open 2022;12:e059647. doi:10.1136/ bmjopen-2021-059647

- Prepublication history and additional supplemental material for this paper are available online. To view these files, please visit the journal online (http://dx.doi.org/10.1136/ bmjopen-2021-059647).

Received 26 November 2021 Accepted 03 February 2022

D Check for updates

(c) Author(s) (or their employer(s)) 2022. Re-use permitted under CC BY-NC. No commercial re-use. See rights and permissions. Published by BMJ.

${ }^{1}$ Neurology, Alfred Health, Melbourne, Victoria, Australia ${ }^{2}$ Neurology, Austin Health, Heidelberg, Victoria, Australia ${ }^{3}$ Department of Neuroscience, Monash University Central

Clinical School, Melbourne, Victoria, Australia

Correspondence to Dr Jason Charles Ray; j.ray@alfred.org.au

\section{ABSTRACT}

Introduction Status migrainosus is a disabling

complication of migraine, which frequently results in hospitalisation. For patients who fail to respond to simple analgesia, triptans and intravenous prochlorperazine or chlorpromazine, there are limited treatment options, and a paucity of high-quality evidence to guide clinical practice. Eptinezumab, an intravenous monoclonal antibody specific for the calcitonin gene-related peptide ligand which achieves maximal plasma concentration immediately following administration and may improve migraines from day one. Intravenous lignocaine is an anaesthetic medication used in treatment of status migrainosus, often requiring prolonged admissions and with potential cardiac adverse events. The aim of this study is to assess the efficacy and safety of eptinezumab in the treatment of status migrainosus in comparison to intravenous lidocaine. Methods and analysis Status migrainosus inpatient treatment with eptinezumab is a randomised, controlled, single-centre clinical trial conducted in a parallel design with an active comparator conducted in Melbourne, Australia. This study randomises forty patients (1:1) to receive either eptinezumab or an infusion of intravenous lignocaine for up to 5 days. It will assess the effect of eptinezumab compared with intravenous lignocaine in aborting status migrainosus, with the primary outcome of time from infusion until resolution of pain. It will explore several secondary measures including change in health resource utilisation, effect on patient reported outcomes of migraine disability and the safety and tolerability of each medication.

Ethics and dissemination This study has been reviewed and approved by the Human Research Ethics Committee of Alfred Health, local reference number 443/21, and all participants will provide informed consent for participation in the trial and dissemination of results.

Trial registration number The trial registration number is ACTRN12621001616864. The results of this study will be disseminated through peer-reviewed journals, conference presentations and social media.

\section{INTRODUCTION}

Migraine is the leading cause of reversible disability in people under 50, affecting 1.3 billion people worldwide. ${ }^{1}$ Accordingly,
Strengths and limitations of this study

- This study is the first controlled trial of eptinezum$\mathrm{ab}$ in the treatment of status migrainosus, and will provide high-quality evidence for the treatment of status migrainosus

- Strengths of this study include its controlled design, which will increase the quality of data on status migrainosus, the use of an active control, which will provide clinically meaningful outcomes and the incorporation of patient-centred and health-economic outcomes.

- Limitations of this study include that it is a singlesite study, the allowance of concomitant medications during the trial period and the paucity of high-quality data to guide statistical power analyses.

the health economic impact of migraine is substantial. Within Australia, headache is the 20th most common cause for admission to hospital with over 2.3 million admissions costing $\$$ A6.8 billion in 2018. ${ }^{2}$

Patients who present to hospital tend to have unremitting migraine attacks for greater then 72 hours that are termed 'status migrainosus'. Within Australia, headache was the 20th most common diagnosis for patients subsequently admitted to hospital, and less then $2 \%$ of emergency department (ED) presentations for headache are for secondary headaches. $^{2}{ }^{3}$ Within the ED they will commonly receive simple analgesia, triptan and either chlorpromazine or prochlorperazine therapy. Current medical practice for second-line therapies includes a low-dose intravenous infusion of an anaesthetic agent; ketamine or lignocaine (lidocaine), which is recommended as a first-line or secondline treatment by $15 \%$ of clinicians surveyed by the American Headache Society. ${ }^{4}$ This is supported by small retrospective case series, however, requires hospitalisation for up to 
5 days, has potential cardiac and neuro-psychiatric side effects and requires cardiovascular monitoring. ${ }^{56}$

On the basis of theUS Preventative Services Task Force Criteria, for patients who have failed triptan and prochlorperazine therapy, none of the current inpatient treatment options for status migrainosus have high-quality evidence. ${ }^{7}$ With a single randomised trial for intravenous dihydroergotamine having been conducted in $1986 .{ }^{8} \mathrm{~A}$ summary of the current standard-of-care options and their evidentiary basis is presented in table 1 .

The pathological role of calcitonin gene-related peptide (CGRP) in migraine and efficacy of its inhibition in both acute and preventative treatment is well established, and the medications have a favourable side effect profile. ${ }^{910}$ Eptinezumab achieves maximal plasma concentration immediately following intravenous administration, and once systemically available, may improve migraine from day one. In a recent randomised trial, eptinezumab was superior to placebo in the treatment of an acute migraine attack. ${ }^{11}$ With a half-life of 27 days, it continues to exert an effect for a significant period of time. ${ }^{12} 13$ Given the immediacy and prolonged efficacy, eptinezumab is likely to be efficacious in the treatment of status migrainosus.

In designing this trial, several considerations have been made. First, the authors considered that a placebocontrolled trial without an active comparator did not represent best practice in the treatment of a prolonged, painful condition. Second, there exists good evidence for the treatment of acute migraine in the ED, however, poor evidence for second-line agents in hospitalised patients when this has failed. Finally, while anaesthetic agents are currently routinely used clinically, they have several drawbacks including potentially prolonged admission and potential cardiac side effects. The study was designed therefore, to not only provide the first high-quality evidence for eptinezumab as a second-line treatment for

\begin{tabular}{|c|c|}
\hline Therapeutic option & Strength of evidence \\
\hline Subcutaneous sumatriptan & $\begin{array}{l}\text { Strong recommendation, } \\
\text { moderate-quality evidence }\end{array}$ \\
\hline Intravenous prochlorperazine & $\begin{array}{l}\text { Strong recommendation, } \\
\text { high-quality evidence }\end{array}$ \\
\hline Intravenous chlorpromazine & $\begin{array}{l}\text { Weak recommendation, } \\
\text { moderate-quality evidence }\end{array}$ \\
\hline Oral NSAIDs & $\begin{array}{l}\text { Strong recommendation, low- } \\
\text { quality evidence }\end{array}$ \\
\hline Intravenous lignocaine & Low-quality evidence, ${ }^{514}$ \\
\hline Intravenous ketamine & Low-quality evidence ${ }^{15}$ \\
\hline
\end{tabular}

Given the lack of substantive evidence of current inpatient therapy, the significant potential side effect profile and health-economic cost both in prolonged hospital admission and representation there is an urgent need for new therapies.

NSAID, Non-steroidal anti-inflammatory drug. status migrainosus, but also demonstrate its efficacy in comparison to an active treatment.

\section{OBJECTIVES}

\section{Study hypothesis}

The primary clinical hypothesis of this study is that treatment with eptinezumab is non-inferior to current standard clinical care (intravenous lignocaine (lidocaine)) in aborting status migrainosus, in patients who have failed or are inappropriate for triptan and intravenous chlorpromazine therapy. The secondary clinical hypothesis is that treatment with eptinezumab will reduce inpatient length of stay, healthcare utilisation at 3 months, and improve patient reported outcome scales related to their migraine disability.

\section{Study objectives}

Primary objective

The primary objective of this study is to evaluate the effect of eptinezumab compare to intravenous lignocaine in aborting status migrainosus.

\section{Secondary objectives}

This study has several secondary objectives, including:

- To explore the effect of eptinezumab compared with lignocaine on the change from baseline in health resource utilisation.

- To evaluate the effect of eptinezumab compared with intravenous lignocaine on patient-reported outcomes of migraine related disability.

- To evaluate the safety and tolerability of eptinezumab in subjects with status migrainosus.

\section{METHODS AND ANALYSIS}

\section{Trial design and study setting}

Status migrainosus inpatient treatment with eptinezumab (SMITE) is a randomised, controlled, single-centre clinical trial conducted in a parallel design with an active comparator. This trial involves patients from the Alfred Hospital, Melbourne, Australia, a tertiary academic hospital, who have been admitted under the neurology team in treatment of status migrainosus. The protocol described in this article is version 1.0 of the SMITE protocol, approved on 26 August 2021 by the local Human Research Ethics Committee (HREC) $(443 / 21)$ and has been registered with the therapeutic goods administration (CT-2021CTN-03 851-1), and Australia and New Zealand Clinical Trial Registry (ACTRN12621001616864).

\section{Eligibility criteria}

Participants who have admitted under the neurology unit of the Alfred Hospital will be eligible to be included if they fulfil the following inclusion and exclusion criteria:

\section{Inclusion criteria}

- Aged 18-65 inclusive at time of presentation to ED. 
- At least a 1-year history of migraine with or without aura as per the International Classification of Headache Disorders, third edition (ICHD-3) criteria.

- Age of participant at the time of migraine onset $<50$ years old.

- An acute migraine attack that has persisted for $\geq 72$ hours as per the ICHD-3 criteria for status migrainosus.

- Ongoing symptoms despite, or contraindication to, triptan and chlorpromazine therapy.

- In the opinion of the investigator and treating doctor, adequate investigation and consideration has been given for secondary causes of headache prior to enrolment.

- Written informed consent obtained from the participant prior to any study-related procedures (online supplemental file 1).

\section{Exclusion criteria}

- History of hemiplegic migraine, cluster headache or other trigeminal autonomic cephalalgia.

- Current concomitant diagnosis of a secondary type of headache.

- Chronic headache with continuous pain lasting more than 3 weeks.

- Headache, which in the opinion of the investigator or delegate requires further investigation for secondary causes of headache.

- Any clinically significant haematological, endocrine, pulmonary, hepatic, gastrointestinal or neurological disease.

- Received an anti-CGRP product within 6 months.

- History of known hepatic disease with potential for hepatic function impairment.

- History of myocardial infarction, stroke, transient ischaemic attack, unstable angina or revascularisation procedure.

- Cardiac arrhythmia.

- Newly diagnosed or uncontrolled hypertension.

- Currently received treatment for another investigational drug or within five half-lives since ending treatment of another investigational drug.

- Clinically significant confounding pain disorder.

- Uncontrolled or untreated major psychiatric condition.

- Body mass index $>39 \mathrm{~kg} / \mathrm{m}^{2}$.

- Women who are pregnant, breast feeding or planning to become pregnant during the study.

- Previous adverse drug reaction to lignocaine or other local anaesthetics.

- History of malignancy (other than non-melanoma skin cancer, fully treated by excision).

- Previously received intravenous lignocaine for status migrainosus.

- Need for contraindicated proarrhythmic or QT prolonging medication contraindicating lignocaine infusion.

\section{EXPERIMENTAL DESIGN}

\section{Interventions}

This study was registered on 26 November 2021, and at the time of submission, has not commenced recruitment. It is anticipated to recruit for a twelve month period from January 2021 to January 2022. A complete schedule of activities is presented in table 2. The patient will have baseline data collected (see below), and undergo randomisation.

On randomisation, forty subjects will be allocated in a 1:1 ratio (figure 1) to receive either eptinezumab $300 \mathrm{mg}$ or placebo infusion, and admitted to hospital to receive intravenous lignocaine following local guidelines (online supplemental file 2) as standard medical therapy (if they received a placebo infusion on randomisation), or a placebo infusion for up to 5 days, or until their migraine is successfully treated.

Patients will remain in hospital for up to 5 days in order to allow the maximum treatment duration of lignocaine infusion, but will be allowed to discharge if they have successfully treated their migraine, as defined as two consecutive Visual Analogue Scales (VAS) $\leq 2$. A phone interview, will be conducted at 30 days, and an in-person follow-up visit will be conducted at 90 days after the administration of the investigational product. A final safety phone-call will occur after 140 days.

\section{Randomisation}

This is a double-blind study. Treatment assignment will be blinded to all subjects, site personnel and the sponsor. Allocation will be determined through the use of a centralised web-based randomisation module, utilising a random number generator in the allocation form. Participants will be randomised in equal numbers to intervention and control arms. A member of the research team not otherwise involved in the study will enter the participants' details into the randomisation system, informing the unblinded research pharmacist of the outcome. All appropriate steps will be taken to maintain the blinding of the research team, statistician and treating clinician, including the use of opaque administration sets.

A subject's allocation will be recorded with the unblinded research pharmacist, and in in a tamperproof envelope. A subject's treatment assignment will be unblinded in two scenarios. In the first scenario, the subject will be unblinded where the knowledge of the treatment is essential for further management or may potentially impact the safety of the subject.

The second scenario is when a subject remains with an untreated headache at the end of the treatment phase (day 5). At this stage, the subject will be unblinded, in order to allow the treating clinician to guide ongoing management. The ongoing management of the patient at this stage will involve part of their routine clinical care, and not considered part of the clinical trial. The subject will continue to have safety follow-up to 140 days. 
Table 2 Schedule of activities

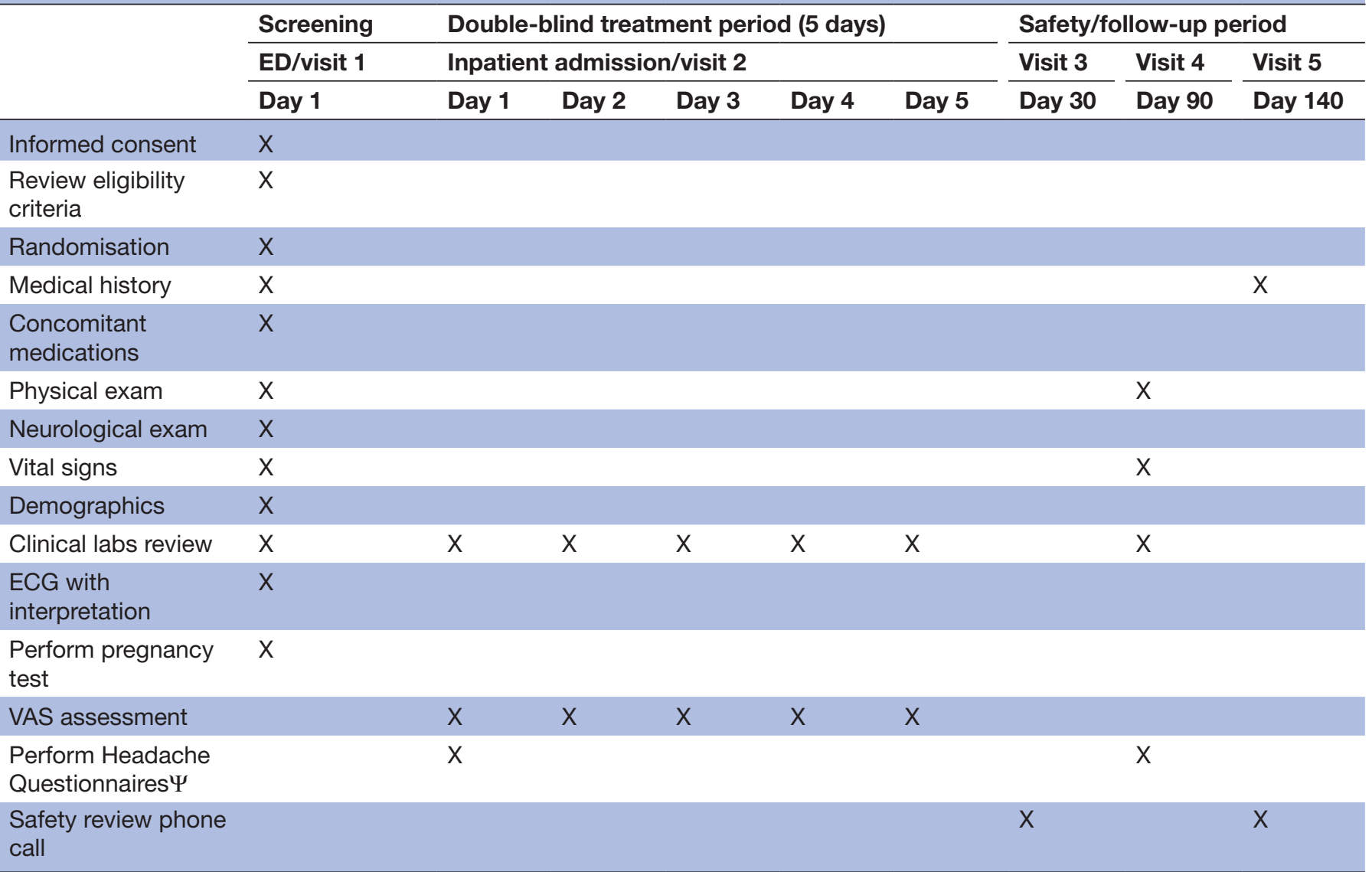

I: headache questionnaires: MIDAS, HIT-6, WPAI, EQ-5D, DASS-21, ESS.

DASS-21, Depression Anxiety Stress Scale; EQ-5D, General Health Status Questionnaire; ESS, Epworth Sleepiness Score; HIT-6, headache impact test; MIDAS, Migraine Disability Scale; VAS, Visual Analogue Scale; WPAI, work productivity and activity impairment.

\section{Concomitant care and interventions}

Throughout the study, investigators may prescribe any concomitant medication or treatment deemed necessary to provide adequate supportive care except for QT prolonging agents and pro-arrhythmic agents. These will be recorded in the case report form, and included in a secondary analysis.

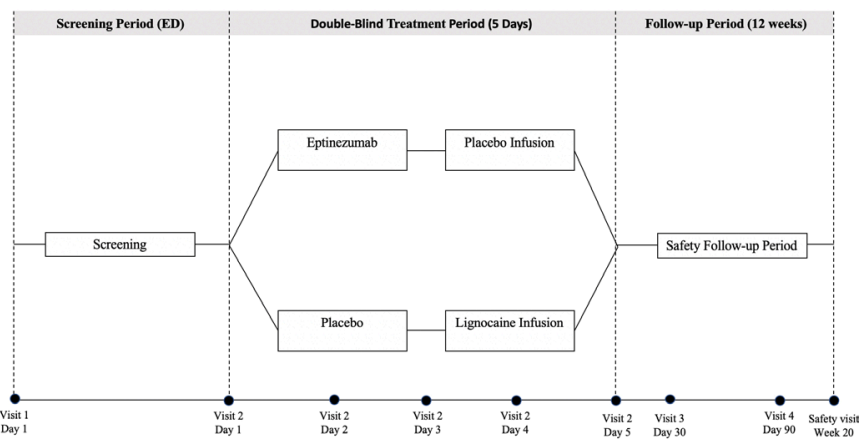

Figure 1 SMITE study schema. ED, emergency department; SMITE, Status migrainosus inpatient treatment with eptinezumab.

\section{Criteria for modifying allocated interventions}

No dose modification of the investigational drug is allowed as part of the study. The non-investigational medication may be modified at the treating clinician's discretion to patient tolerability, in keeping with current clinical practice (see online supplemental file 2).

\section{Data collection and management}

At the time of enrolment, we will record baseline demographic data, a complete medical and headache history, physical examination, baseline ECG and pregnancy and routine blood tests, as well as baseline efficacy data. While admitted in hospital, patients will undergo efficacy and safety assessments as outlined below. Patients will then be contacted at day 30, 90 and 140 for safety and efficacy follow-up. Data will be recorded in a secure hospital server, with range and validity checks for data values, and double data entry.

\section{Efficacy data}

In addition to 3 hourly VAS scores while awake, patients will undergo the following assessment:

- Date and time of start of current migraine.

- Date and time of resolution of current migraine (defined as two consecutive VAS $\leq 2$ ). 
- Date and time of admission, interventional product infusion and discharge from hospital.

- Baseline monthly number of primary healthcare provider and ED visits for migraine.

- Monthly number of primary healthcare provider and ED visits for migraine at 90 days.

- Baseline score of Migraine Disability Scale (MIDAS), Headache impact test (HIT-6), work productivity and activity impairment (WPAI) and General Health Status (EQ-5D) questionnaires.

- Score of MIDAS, HIT-6, WPAI and EQ-5D at day 90.

\section{Safety data}

As part of the routine safety assessment of patients receiving lignocaine infusion in hospital, all patients will receive 4 hourly vital sign observation and daily ECGs and blood tests to assess for electrolyte abnormalities while admitted.

All adverse events will be collected until 90 days after the last dose of the study regardless of whether or not the participant received the study intervention, and as observed or reported by study participants. Care will be taken not to introduce bias when detecting adverse events by using open-ended non-leading verbal questioning of the participant.

\section{Outcomes}

The primary endpoint for the study will be the time from infusion initiation to discharge. A number of secondary endpoints will be explored in order to meet the objectives of the study, summarised in table 3 . The primary endpoint of the study, time to symptom resolution has been chosen both as a marker of the efficacy of eptinezumab, and to demonstrate the health-economic and patient-centred outcomes of duration of symptoms (and consequently burden of length of stay in hospital).

\section{Participant timeline}

The total participant timeline is presented in the schedule of activities (table 2). This incorporates hospital admission for up to 5 days post enrolment, secondary endpoints at 90 days and safety-follow up data to 140 days.

\section{Patient and public involvement}

Members of the public or patients were not involved in the study design.

\section{STATISTICAL CONSIDERATIONS Statistical hypothesis}

As this is a non-inferiority study, the primary null hypothesis is that the efficacy of eptinezumab $300 \mathrm{mg}$ intravenous infusion measured in hazard rate of discharge is equal to or worse than non-inferiority margin to intravenous lignocaine in treating status migrainosus. The alternative hypothesis is that the efficacy of eptinezumab 300 $\mathrm{mg}$ intravenous infusion compare to intravenous lignocaine in treating status migrainosus is higher than the non-inferiority margin.

- H0: $\mathrm{b} \leq \delta$ (Inferior).

- H1: $\mathrm{b}>\delta$ (non-inferior).

Table 3 SMITE study objectives and end-points

Objectives Endpoints

To evaluate the effect of eptinezumab compared with intravenous lignocaine in aborting status migrainosus
Time from infusion to discharge (Primary outcome)

- Duration of symptoms post infusion

- Visual Analogue Scale (VAS) on discharge or at day five (primary outcome)

- Change in VAS from admission to discharge (primary outcome)

- Use of rescue therapies during the 5-day admission (primary outcome)

- Pain freedom at 2 hours (secondary outcome)

- Freedom from most bothersome symptom at 2 hours (secondary outcome)

- Sustained pain freedom at 24 hours postinfusion in patients who achieve pain freedom (secondary outcome)

To explore the effect of eptinezumab compared with lignocaine on the change from baseline in health resource utilisation

- Change from baseline in monthly number of primary healthcare provider visits (secondary outcome)

- Change from baseline in monthly emergency department visits (secondary outcome)

$\begin{array}{ll}\text { To evaluate the effect of eptinezumab compared } & \text { Change from baseline in MIDAS (secondary outcome) } \\ \text { with intravenous lignocaine on patient reported } & \text { Change from baseline HIT-6 (secondary outcome) } \\ \text { outcomes of migraine related disability } & \text { Change from baseline in WPAI (secondary outcome) } \\ & \text { Change from baseline in EQ-5D (secondary outcome) } \\ \begin{array}{l}\text { To evaluate the safety and tolerability of } \\ \text { eptinezumab in subjects with status migrainosus }\end{array} & \text { Clinerse events (other safety outcome) }\end{array}$

EQ-5D, General Health Status Questionnaire; HIT6, headache impact test; MIDAS, Migraine Disability Scale; SMITE, Status migrainosus inpatient treatment with eptinezumab; WAPI, work productivity and activity impairment. 
Where $b$ is the coefficient equivalent to the log-HR and $\delta$ is the non-inferiority margin and $<0$.

For efficacy analyses, data will be analysed according to participants' randomisation assignments, according to an intention to treat analysis. For safety data analyses, the participants will be analysed according to actual treatment received.

\section{Sample size}

There is a paucity of high-quality clinical data on the efficacy of either the experimental or control group in the treatment of status migrainosus, with the largest case series of lignocaine including only five patients with status migrainosus. As such a study of 40 (forty) patients total is proposed. Using one-sided significance level of 0.025 , for the primary outcome, the sample size will provide $80 \%$ power for a non-inferiority margin of $\delta=-0.89$ or HR of 0.41 , that is, the hazard rate of discharge in the eptinezumab group is $41 \%$ or lower of the lignocaine group, in univariable Cox regression.

In subsequent secondary multivariable analysis, adjustment for potential risk factors associated with treatment outcome, that is, baseline headache frequency and composite risk score, will be considered. These factors are conservatively assumed to have moderate to strong association $\left(\mathrm{R}^{2}=0.5\right)$ with the treatment group, so $50 \%$ of the variance of the primary predictor is explained by these factors. We estimate to have $80 \%$ power for a noninferiority margin of $\delta=-1.25$ or $\mathrm{HR}=0.29$ in multivariable Cox regression after adjustment for these factors. Given the short treatment period, we anticipate there will be no drop-outs during the treatment phase.

\section{Statistical analysis}

Data analysis will be blinded. The efficacy analyses will be based on an intention to treat analysis. The primary efficacy outcome, time to discharge, will be summarised using median and IQRs as it is anticipated to be nonnormally distributed. Univariable Cox regression will be used to estimate the HR of discharge between the eptinezumab and lignocaine groups and its $95 \%$ CI will be reported which corresponds to a one-sided significance level of 0.025 . If the upper limit of the $95 \%$ CI is lower than the non-inferiority margin of $\mathrm{HR}=0.41$, this would indicate eptinezumab has an unacceptably worse efficacy than lignocaine. Non-inferiority p value will also be calculated. Multivariable Cox regression with adjustment of baseline headache frequency and composite risk score as subsequent secondary analysis will be performed.

For secondary efficacy and follow-up outcomes, continuous variables will be summarised using means and SD or medians and IQRs depending on the underlying data distribution. Categorical variables will be expressed as counts and proportions. Exploratory univariable analysis will be conducted using Student's t-test, Mann-Whitney U test, $\chi^{2}$ or Fisher's exact tests where appropriate.

Safety and tolerability outcomes, such as adverse events, will be descriptively summarised.
All statistical tests will be performed by using statistical software packages R (R Core Team), Stata (StataCorp) or SPSS (IBM).

\section{Auditing}

An annual audit detailing recruitment, adverse events and complaints will be performed and submitted to the local HREC, in compliance with local policies. This will be performed by the principal investigator. An interim analysis will not be performed due to the small sample size and projected length of the study.

\section{ETHICS AND DISSEMINATION}

\section{Research ethics approval and consent}

This study will be conducted in accordance with the ICH note for guidance on Good Clinical Practice (GCP) and has been reviewed and approved by the Human Research Ethics Committee of Alfred Health (local reference number 443/21). Informed consent will be obtained from potential participants by a senior doctor in the trial team, who has GCP certification and is registered with the local HREC, for participation in the trial and dissemination of trial results. The consent form is included as online supplemental file 1 .

\section{Confidentiality and dissemination}

Personal information will be stored in a study database on a secured hospital server, accessible only to the study team. Results will occur only in a deidentified, grouped manner to reduce the risk of identification of participants. The results will be actively disseminated through peer-reviewed journals, conference presentations and social media.

\section{Data sharing}

Requests for deidentified individual data, and the study protocol can be made between 3 months and 5 years following publication by researchers with a methodologically sound proposal. Requests can be made by arrangement with the corresponding author via j.ray@alfred.org. au.

\section{DISCUSSION \\ Strengths and limitations of the SMITE trial design}

This study is the first randomised controlled study to evaluate the use of eptinezumab in the treatment of status migrainosus. Its design, in comparing eptinezumab to existing standard treatment, will provide clinically a meaningful outcome for clinicians. In addition, it incorporates both patient-centred and health-economic outcomes to provide a greater context of the efficacy of the trial medication.

There are several potential limitations to this study design. As a single-site study, the generalisability of the results to diverse patient populations will require careful consideration. The allowance of concomitant medications during the trial period may have the effect of diluting the 
treatment difference between patient groups, however, was felt to be important ethically in patients hospitalised for a painful condition by the investigators. The paucity of high-quality data on the efficacy of lignocaine also limits the power analysis. Finally, this study will be undertaken in the midst of the COVID-19 pandemic. It is likely that hospital resources will be stretched, and hospital presentations for non-respiratory illnesses will be reduced. It is anticipated that this may cause slower recruitment then projected, however, should not interfere with the study.

Twitter Jason Charles Ray @dr_jray1 and Elspeth J Hutton @spiraldance1

Authors contributions JCR and EJH conceptualised the project and developed the protocol with the assistance of JG and GR. ZC provided additional expertise in the statistical analysis. JCR drafted the manuscript and all authors reviewed and edited the manuscript.

Funding for this study is supported by Lundbeck.

Competing interests JCR has received compensation from the Pharmaceutical Society of Australia, sponsored by Viatris for educational material. ZC is supported by an Early Career Fellowship from the National Health and Medical Research Council (NHMRC) of Australia (GNT1156444). He/his institution has received consultancy fees and/or research grants from Arvelle Therapeutics and UCB Pharma for works outside the submitted work. EJH has served on advisory boards for Sanofi-Genzyme, Novartis, Teva, Eli Lilly, Allergan, Lundbeck, been involved in clinical trials sponsored by Novartis, Teva, Xalud, Daewong and Novotech, and has received payment for educational presentations from Allergan, Teva, Eli Lilly and Novartis.

Patient consent for publication Not applicable.

Provenance and peer review Not commissioned; externally peer reviewed.

Supplemental material This content has been supplied by the author(s). It has not been vetted by BMJ Publishing Group Limited (BMJ) and may not have been peer-reviewed. Any opinions or recommendations discussed are solely those of the author(s) and are not endorsed by BMJ. BMJ disclaims all liability and responsibility arising from any reliance placed on the content. Where the content includes any translated material, BMJ does not warrant the accuracy and reliability of the translations (including but not limited to local regulations, clinical guidelines, terminology, drug names and drug dosages), and is not responsible for any error and/or omissions arising from translation and adaptation or otherwise.

Open access This is an open access article distributed in accordance with the Creative Commons Attribution Non Commercial (CC BY-NC 4.0) license, which permits others to distribute, remix, adapt, build upon this work non-commercially, and license their derivative works on different terms, provided the original work is properly cited, appropriate credit is given, any changes made indicated, and the use is non-commercial. See: http://creativecommons.org/licenses/by-nc/4.0/.

\section{ORCID iD}

Jason Charles Ray http://orcid.org/0000-0003-4833-5507

\section{REFERENCES}

1 Steiner TJ, Stovner LJ, Vos T, et al. Migraine is first cause of disability in under 50S: will health politicians now take notice? J Headache Pain 2018;19:17-20.

2 Economics DA. Migraine in Australia Whitepaper, 2018. Available: https://www2.deloitte.com/au/en/pages/economics/articles/ migraine-australia-whitepaper.html

3 Doretti A, Shestaritc I, Ungaro D, et al. Headaches in the emergency department -A survey of patients' characteristics, facts and needs. J Headache Pain 2019;20:100.

4 Dougherty C. Emergency and inpatient treatment of migraine: an American headache Society survey. $\mathrm{Br} J$ Med Med Res 2014;4:3800-13.

5 Ayulo MA, Phillips KE, Tripathi S. Safety and efficacy of IV lidocaine in the treatment of children and adolescents with status migraine. Pediatr Crit Care Med 2018;19:755-9.

6 Gil-Gouveia R, Goadsby PJ. Neuropsychiatric side-effects of lidocaine: examples from the treatment of headache and a review. Cephalalgia 2009;29:496-508.

7 Orr SL, Aubé M, Becker WJ, et al. Canadian headache Society systematic review and recommendations on the treatment of migraine pain in emergency settings. Cephalalgia 2015;35:271-84.

8 Raskin $\mathrm{NH}$. Repetitive intravenous dihydroergotamine as therapy for intractable migraine. Neurology 1986;36:995.

9 Edvinsson L, Haanes KA, Warfvinge K, et al. CGRP as the target of new migraine therapies - successful translation from bench to clinic. Nat Rev Neurol 2018;14:338-50.

10 Ray JC, Kapoor M, Stark RJ, et al. Calcitonin gene related peptide in migraine: current therapeutics, future implications and potential off-target effects. J Neurol Neurosurg Psychiatry 2021;92:1325-34.

11 Winner PK, McAllister P, Chakhava G, et al. Effects of intravenous Eptinezumab vs placebo on headache pain and most Bothersome symptom when initiated during a migraine attack: a randomized clinical trial. JAMA 2021;325:2348-56.

12 Lipton RB, Goadsby PJ, Smith J, et al. Efficacy and safety of eptinezumab in patients with chronic migraine. Neurology 2020;94:e1365-77.

13 Baker B, Schaeffler B, Beliveau M, et al. Population pharmacokinetic and exposure-response analysis of eptinezumab in the treatment of episodic and chronic migraine. Pharmacol Res Perspect 2020;8.

14 Hand PJ, Stark RJ. Intravenous lignocaine infusions for severe chronic daily headache. Med J Aust 2000;172:157-9.

15 Lauritsen C, Mazuera S, Lipton RB, et al. Intravenous ketamine for subacute treatment of refractory chronic migraine: a case series. $J$ Headache Pain 2016;17:106. 